\title{
Models for da Costa's paraconsistent set theory
}

\author{
Aldo Figallo Orellano ${ }^{1,2}$ and Juan Sebastián Slagter ${ }^{1}$ \\ ${ }^{1}$ Departamento de Matemática - Universidad Nacional del Sur (UNS) \\ Bahía Blanca - Buenos Aires - Argentina \\ ${ }^{2}$ Centre for Logic, Epistemology and The History of Science (CLE) \\ University of Campinas (Unicamp) - Brazil \\ aldofigallo@gmail.com, juan.slagter@uns.edu.ar
}

\begin{abstract}
In this work we will be constructed F-structures-valued models as generalization of Boolean-valued models and proved that these models that verify Leibniz' Law validate all the set-theoretic axioms of da Costa's Paraconsistent Set Theory.
\end{abstract}

Resumo. Neste trabalho vamos construir os modelos de F-estruturas valuadas como uma generalização dos modelos Booleanos valuados e provaremos que esses modelos que verificam a Lei de Leibniz validam todos os axiomas conjuntistas da Teoria Paraconsistente de Conjunto de da Costa..

\section{Introduction and Preliminaries}

The publication in 1963 of da Costa's Habilitation thesis Sistemas Formais Inconsistentes constitutes a landmark in the history of paraconsistency. In that thesis, da Costa introduced the hierarchy $C_{n}$ (for $n \geq 1$ ) and $C_{\omega}$ of $C$-systems [da Costa 1963]. Recall that Paraconsistency is the study of logic systems having a negation $\neg$ which is not explosive, that is, there exist formulas $\alpha$ and $\beta$ in the language of the logic such that $\beta$ is not derivable from the contradictory set $\{\alpha, \neg \alpha\}$.

Now, consider the signature $\Sigma=\{\rightarrow, \wedge, \vee, \neg\}$ and recall that $C_{\omega}$ is defined over the language $\mathcal{L}_{\Sigma}$ determined by the Hilbert calculus from axiom schemas from Intuitionistic Positive Calculus, the rule modus ponens and the following axiom schemas: $(C \omega 1)$ $\alpha \vee \neg \alpha$ and $(C \omega 2) \neg \neg \alpha \rightarrow \alpha$. We are going to consider the usual notion of derivation of a formula $\alpha$ form $\Gamma$ in $C_{\omega}$ and we denote by $\Gamma \vdash \alpha$.

Later, da Costa visited Universidad Nacional del sur in 1969 and proposed Fidel to find a semantics for $C_{n}$ and $C_{\omega}$. In that time, they knew the negation $\neg$ was not congruencial. In fact, as we are going to see, $C_{\omega}$ is not algebrizable with Blok-Pigozzi's method. Fidel jumped this difficulty by means of a presentation of a novel algebraic-relational 
class of structures called $F$-structure by adapting Lindenbaum-Tarski method in order to prove soundness and completeness theorem.

Now, recall that an algebra $\mathcal{A}=\langle A, \vee, \wedge, \rightarrow, 0,1\rangle$ is said to be a Heyting algebra if the reduct $\langle A, \vee, \wedge, 0,1\rangle$ is a bounded distributive lattice and the condition $x \wedge y \leq z$ iff $x \leq y \rightarrow z(*)$ holds. Besides, the algebra $\mathcal{A}=\langle A, \vee, \wedge, \rightarrow, 1\rangle$ is said to be generalized Heyting algebra if the reduct $\mathcal{A}=\langle A, \vee, \wedge\rangle$ it is a distributive lattice and $*$ is verified.

Recall, that a $C_{\omega}$-structure ([Fidel 1977]) is a system $\left\langle\mathbf{A},\left\{N_{x}\right\}_{x \in A}\right\rangle$ where $\mathbf{A}$ is a generalized Heyting algebra and $\left\{N_{x}\right\}_{x \in A}$ is a family of subset of $A$ such that the following conditions hold for every $x \in A$ :

(i) $x \vee x^{\prime}=1$ for each $x^{\prime} \in N_{x}$,

(ii) for every $x^{\prime} \in N_{x}$ there is $x^{\prime \prime} \in N_{x^{\prime}}$ such that $x^{\prime \prime} \leq x$.

Besides, we say that a function $v: \mathfrak{F m} \rightarrow\left\langle\mathbf{A},\left\{N_{x}\right\}_{x \in A}\right\rangle$ is a $C_{\omega}$-valuation (or simply valuation) if the following conditions hold:

(v1) $v(\alpha) \in A$ where $\alpha$ is atomic formula,

(v2) $v(\alpha \# \beta)=v(\alpha) \# v(\beta)$ where $\# \in\{\wedge, \vee, \rightarrow\}$,

(v3) $v(\neg \alpha) \in N_{v(\alpha)}$ and $v(\neg \neg \alpha) \leq v(\alpha)$.

For us a formula $\alpha$ will be semantically valid if for all valuation $v$ and every $C_{\omega^{-}}$ structure $\left\langle\mathbf{A},\left\{N_{x}\right\}_{x \in A}\right\rangle, v(\alpha)=1$ and then, we denote $\vDash \alpha$. Moreover, for a given set of formulas $\Gamma$, it is said that $\alpha$ is a semantic consequence of $\Gamma$, and we write $\Gamma \vDash \alpha$ if for every valuation $v$ and every $C_{\omega}$-structure $\left\langle\mathbf{A},\left\{N_{x}\right\}_{x \in A}\right\rangle$, if $v(\beta)=1$ for every $\beta \in \Gamma$ then $v(\alpha)=1$.

Theorem 1.1 ([Fidel 1977]) Let $\Gamma \cup\{\alpha\}$ be a set of formulas of $C_{\omega}$. Then, $\Gamma \vdash \alpha$ if only if $\Gamma \vDash \alpha$.

In order to see that $C_{\omega}$ is paraconsistent logic, we are going to consider the structure $\mathcal{M}_{3}=\left\langle H_{3}, N_{0}=\{1\}, N_{\frac{1}{2}}=\{1\}, N_{1}=\{0,1\}\right\rangle$ where $H_{3}=\left(\left\{0, \frac{1}{2}, 1\right\}, \wedge, \vee, \rightarrow\right.$ $, 0,1$ ) is a 3 -valued Gödel algebra (or 3-valued Heyting algebra). Thus, is is clear that $\not \forall(\neg \alpha \wedge \alpha) \rightarrow \beta$ for all $\alpha, \beta$. To see this, it is enough to consider a valuation $v$ such that $v(\alpha)=\frac{1}{2}, v(\neg \alpha)=1$ and $v(\beta)=0$. So, from Theorem 1.1, we have $\forall(\neg \alpha \wedge \alpha) \rightarrow \beta$ and taking into account the (meta-)deduction theorem, we have $\{\neg \alpha, \alpha\} \forall \beta$.

\section{The $\boldsymbol{F}$-structure-valued models for paraconsistent set theory}

In [L-T 2015], Löwe and Taradfer proposed a 3-valued model for the negation-free fragment of a paraconsistent set theory based on the idea of boolean-valued models for Set Theory, see [Bell 2005]. In this work, we are going to present full models for a paraconsistent set theory. To this end, we fix a model of set theory $\mathrm{V}$ and a completed $C_{\omega}$-structure 
$\langle\mathbf{A}, N\rangle$. Let us construct a universe of names by transfinite recursion:

$$
\begin{gathered}
\mathbf{V}_{\xi}^{\langle\mathbf{A}, N\rangle}=\left\{x: x \text { a function and } \operatorname{ran}(x) \subseteq A \text { and } \operatorname{dom}(x) \subseteq \mathbf{V}_{\zeta}^{\langle\mathbf{A}, N\rangle} \text { for some } \zeta<\xi\right\} \\
\mathbf{V}^{\langle\mathbf{A}, N\rangle}=\left\{x: x \in \mathbf{V}_{\xi}^{\langle\mathbf{A}, N\rangle} \text { for some } \xi\right\}
\end{gathered}
$$

The class $\mathbf{V}^{\langle\mathbf{A}, N\rangle}$ is called the $C_{\omega}$-structure-valued model over $\langle\mathbf{A}, N\rangle$. Let us observe that we only need the set $A$ in order to define $\mathbf{V}_{\xi}\langle\mathbf{A}, N\rangle$. By $\mathcal{L}_{\in}$, we denote the first-order language of set theory which consists of only the propositional connectives $\{\rightarrow, \wedge, \vee, \neg\}$ of the $C_{\omega}$ and two binary predicates $\in$ and $\approx$. We can expand this language by adding all the elements of $\mathbf{V}^{\langle\mathbf{A}, N\rangle}$; the expanded language we will denote $\mathcal{L}_{\langle\mathbf{A}, N\rangle}$.

Induction principles. The sets

$$
\mathbf{V}_{\zeta}=\left\{x: x \subseteq \mathbf{V}_{\xi}, \text { for some } \xi<\zeta\right\}
$$

are definable for every ordinal $\xi$ and then, every set $x$ belongs to $\mathbf{V}_{\alpha}$ for some $\alpha$. So, this fact induce a function $\operatorname{rank}(x)=$ least ordinal $\xi$ such that $x \in \mathbf{V}_{\xi}$. Since $\operatorname{rank}(x)<$ $\operatorname{rank}(y)$ is well-founded we induce a principle of induction on rank: let $\Psi$ be a property over sets. Assume for every set $x$, if $\Psi(y)$ holds for every $y$ such that $\operatorname{rank}(y)<\operatorname{rank}(x)$, then $\Psi(x)$ holds. Thus, $\Psi(x)$ for every $x$.

From the latter, Induction Principles (IP) holds in $\mathbf{V}^{\langle\mathbf{A}, N\rangle}$. Assume for every $x \in$ $\mathbf{V}^{\langle A, N\rangle}$, if $\Psi(y)$ holds for every $y \in \operatorname{dom}(x)$ then $\Psi(x)$ holds. Hence, $\Psi(x)$ holds for every $x \in \mathbf{V}^{\langle\mathbf{A}, N\rangle}$.

By simplicity, we note every set $u \in \mathbf{V}^{\langle\mathbf{A}, N\rangle}$ by its name $u$ of $\mathcal{L}_{\langle\mathbf{A}, N\rangle}$. Besides, we will write $\varphi(u)$ instead of $\varphi(x / u)$. Now, we are going to define a valuation by induction on the complexity of a closed formula in $\mathcal{L}_{\langle\mathbf{A}, N\rangle}$. Indeed,

Definition 2.1 For a given complete $C_{\omega}$-structure $\mathfrak{A}=\langle\mathbf{A}, N\rangle$, the mapping $\|\cdot\|^{\mathfrak{A}}$ : $\mathcal{L}_{\langle\mathbf{A}, N\rangle} \rightarrow\langle\mathbf{A}, N\rangle$ is defined as follow:

$$
\begin{aligned}
& \|u \in v\|^{\mathfrak{A}}=\bigvee_{x \in \operatorname{dom}(v)}\left(v(x) \wedge\|x \approx u\|^{\mathfrak{A}}\right), \\
& \left.\|u \approx v\|^{\mathfrak{A}}=\bigwedge_{x \in \operatorname{dom}(u)}(u(x)) \rightarrow\|x \in v\|^{\mathfrak{A}}\right) \wedge \bigwedge_{x \in \operatorname{dom}(v)}\left(v(x) \rightarrow\|x \in u\|^{\mathfrak{A}}\right), \\
& \|\varphi \# \psi\|^{\mathfrak{A}}=\|\varphi\|^{\mathfrak{A}} \tilde{\#}\|\psi\|^{\mathfrak{A}} \text {, for every } \# \in\{\wedge, \vee, \rightarrow\} \\
& \|\neg \alpha\|^{\mathfrak{A}} \in N_{\|\alpha\|^{\mathfrak{A}}} \text { and }\|\neg \neg \alpha\|^{\mathfrak{A}} \leq\|\alpha\|^{\mathfrak{A}}, \\
& \|\exists x \varphi\|^{\mathfrak{A}}=\bigvee_{u \in \mathbf{V}^{\langle\mathbf{A}, N\rangle}}\|\varphi(u)\|^{\mathfrak{A}} \text { and }\|\forall x \varphi\|^{\mathfrak{A}}=\bigwedge_{u \in \mathbf{V}^{\langle\mathbf{A}, N\rangle}}\|\varphi(u)\|^{\mathfrak{A}} .
\end{aligned}
$$

$\|\varphi\|^{\mathfrak{A}}$ is called the truth-value of the sentence $\varphi$ in the language $\mathcal{L}_{\langle\mathbf{A}, N\rangle}$ in the $C_{\omega}$-structure-valued model over $\langle\mathbf{A}, N\rangle$.

In the in the book [Rasiowa 1974], it was presented a algebraic semantics for certain first-order algebraizable logics. Following this algebraic treatment of this systems, 
we define, for every formula $\alpha(x),\|\alpha(x / u)\|^{\mathfrak{A}}=\|\alpha(u)\|^{\mathfrak{A}}$ for any $u \in \mathbf{V}$. It is clear that we can prove this condition for negation-free formulas but for formulas with negation, we need to require it as axiom. This condition is well-known as substitution lemma.

Definition 2.2 A sentence $\varphi$ in the language $\mathcal{L}_{\langle\mathbf{A}, N\rangle}$ is said to be valid in $\mathbf{V}^{\langle\mathbf{A}, N\rangle}$, which is denoted by $\mathbf{V}^{\langle\mathbf{A}, N\rangle} \vDash \varphi$, if $\|\varphi\|^{\mathfrak{A}}=1$.

For the sake of simplicity, we write $\|\varphi\|$ instead of $\|\varphi\|^{\mathfrak{A}}$. Besides, it is clear that every completed $C_{\omega}$-structure $\langle\mathbf{A}, N\rangle$, the element $\bigwedge x$ is the first element of $A$ and so A is a complete Heyting algebra. We denote by " 0 " this element. Finally, it is worth mentioning that for every closed formula $\phi$ of $\mathcal{L}_{\langle\mathbf{A}, N\rangle}$, we have $\|\phi\| \in A$. Then, the following lemma has the same proof that the case of intuitionistic set theory.

Lemma 2.3 For a given completed $C_{\omega}$-structure $\langle\mathbf{A}, N\rangle$. Then, $\|u \approx u\|=1, u(x) \leq$ $\|x \in u\|$ for every $x \in \operatorname{dom}(u)$, and $\|u=v\|=\|v=u\|$, for every $u, v \in \mathbf{V}^{\langle\mathbf{A}, N\rangle}$.

In the classical and intuitionistic set theory, we have that the manes represent objects and if we have equivalent objects they would have to have the same properties. This is known as indiscernibility of identicals and it could be considered as Leibniz's law by the following axiom:

$$
u \approx v \wedge \varphi(u) \rightarrow \varphi(v)
$$

In the next, we are going to consider complete $C_{\omega}$-structures which verify the Leibniz's law. It is important to note that we have $C_{\omega}$-structures that verify this law, it is enough to require $1 \in N_{x}$ for all $x \in A$ for every $x \neq 1$ and $0 \in N_{1}$.

We will adopt the following notation, for every formula $\varphi(x)$ and every $u \in$ $\mathbf{V}^{\langle\mathbf{A}, N\rangle}$ :

$$
\exists x \in u \varphi(x)=\exists x(x \in u \wedge \varphi(x))
$$

and

$$
\forall x \in u \varphi(x)=\forall x(x \in u \rightarrow \varphi(x))
$$

Thus, we have the following

Lemma 2.4 Let $\langle\mathbf{A}, N\rangle$ be a complete Leibniz $C_{\omega}$-structure, for every formula $\varphi(x)$ and every $u \in \mathbf{V}^{\langle\mathbf{A}, N\rangle}$ we have

$$
\begin{aligned}
& \|\exists x \in u \varphi(x)\|=\bigvee_{x \in \operatorname{dom}(u)}(u(x) \wedge\|\varphi(x)\|), \\
& \|\forall x \in u \varphi(x)\|=\bigwedge_{x \in \operatorname{dom}(u)}(u(x) \rightarrow\|\varphi(x)\|) .
\end{aligned}
$$




\subsection{Paraconsistent Set Theory}

The basic system of paraconsistent set theory here is called $Z F C_{\omega}$ and consists of first order version $\mathcal{Q} C_{\omega}$ of $C_{\omega}$ over the first-order signature $\Theta_{\omega}$ which contains an equality predicate $=$ and a binary predicate $\epsilon$.

Definition 2.5 The system $Z F C_{\omega}$ is the first order theory with equality obtained from the logic $\mathcal{Q} C_{\omega}$ over $\Theta_{\omega}$ by adding the following set-theoretic axiom schemas:

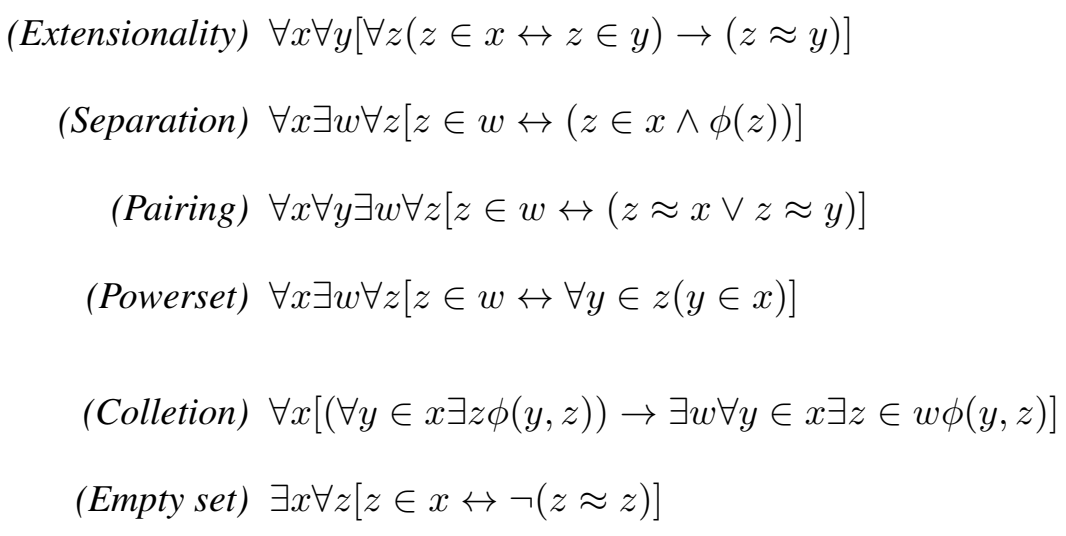

The set satisfying this axiom is, by extensionality, unique and we refer to it with $\emptyset$.

$$
\begin{aligned}
& \text { (Union) } \forall x \exists w \forall z[z \in w \leftrightarrow \exists y \in x(z \in y)] \\
& \text { (Infinity) } \exists x\left[\emptyset \in x \wedge \forall y \in x\left(y^{+} \in x\right)\right]
\end{aligned}
$$

From union and pairing and extensionality, we can note by $y^{+}$the unique set $y \cup\{y\}$.

(Induction) $\forall x[(\forall y \in x \phi(y)) \rightarrow \phi(x)] \rightarrow \forall x \phi(x)$.

The last nine axioms are usually used to define Intuitionistic Zermelo-Fraenkel set theory (IZF); see for instance, [Bell 2014]. Now, we are going to prove that Leibniz $C_{\omega}$-structurevalued models are models for a paraconsistent set theory. Indeed,

Definition 2.6 Let $\langle\mathbf{A}, N\rangle$ be a complete $C_{\omega}$-substructure. Given collection of sets $\left\{u_{i}\right.$ : $i \in I\} \subseteq \mathbf{V}^{\langle\mathbf{A}, N\rangle}$ and $\left\{a_{i}: i \in I\right\} \subseteq A$, the mixture $\Sigma_{i \in I} a_{i} \cdot u_{i}$ is the fucntion $u$ with $\operatorname{dom}(u)=\bigcup_{i \in I} \operatorname{dom}\left(u_{i}\right)$ and $u(x)=\bigvee_{i \in I} a_{i} \wedge\left\|x \in u_{i}\right\|$.

Lemma 2.7 (Mixing Lemma) Let $u$ be the mixture $\Sigma_{i \in I} a_{i} \cdot u_{i}$. If $a_{i} \wedge a_{j} \leq\left\|u_{i}=u_{j}\right\|$ for all $i, j \in I$, then $a_{i} \leq\left\|u_{i}=u\right\|$.

A set $B$ refines a set $A$ if for all $b \in B$ there is some $a \in A$ such that $b \leq a$. A Heyting algebra $H$ is refinable if every subset $A \subseteq H$ there exists some anti-chaim $B$ in $H$ that refines $A$ and verifies $\bigvee A=\bigvee B$. 
Theorem 2.8 Let $\langle\mathbf{A}, N\rangle$ be a complete $C_{\omega}$-substructure such that $A$ is refinable. If $\mathbf{V}^{\langle\mathbf{A}, N\rangle} \vDash \exists x \psi(x)$, then there is $u \in \mathbf{V}^{\langle\mathbf{A}, N\rangle}$ such that $\mathbf{V}^{\langle\mathbf{A}, N\rangle} \vDash \psi(u)$.

Now, given a complete $C_{\omega}$-substructure $\left\langle\mathbf{A}^{\prime}, N^{\prime}\right\rangle$ of $\langle\mathbf{A}, N\rangle$, we have the associated models $\mathbf{V}^{\left\langle\mathbf{A}^{\prime}, N^{\prime}\right\rangle}$ and $\mathbf{V}^{\langle\mathbf{A}, N\rangle}$. Then, it is easy to see that $\mathbf{V}^{\left\langle\mathbf{A}^{\prime}, N^{\prime}\right\rangle} \subseteq \mathbf{V}^{\langle\mathbf{A}, N\rangle}$.

On the other hand, we say that a formula $\psi$ is restricted if all quantifiers are of the form $\exists y \in x$ or $\forall y \in x$, then we have

Lemma 2.9 For any complete $C_{\omega}$-substructure $\left\langle\mathbf{A}^{\prime}, N^{\prime}\right\rangle$ of $\langle\mathbf{A}, N\rangle$ and any restricted formula $\psi\left(x_{1}, \cdots, x_{n}\right)$ with variables in $\mathbf{V}^{\left\langle\mathbf{A}^{\prime}, N^{\prime}\right\rangle}$ the equality $\left\|\psi\left(x_{1}, \cdots, x_{n}\right)\right\|^{\left\langle\mathbf{A}^{\prime}, N^{\prime}\right\rangle}=$ $\left\|\psi\left(x_{1}, \cdots, x_{n}\right)\right\|^{\langle\mathbf{A}, N\rangle}$.

Next, we are going to consider the Boolean algebra $2=(\{0,1\}, \wedge, \vee, \neg, 0,1)$ and the natural mapping $\hat{\imath}: \mathbf{V}^{\langle A, N\rangle} \rightarrow \mathbf{V}^{\left\langle\mathbf{2}, N_{\mathbf{2}}\right\rangle}$ where $N_{\mathbf{2}}=\{(0,1),(1,0)\}$ defined by $\hat{u}=\{\langle\hat{v}, 1\rangle: v \in u\}$. This is well defined by recursion on $v \in \operatorname{dom}(u)$. It is clear that $\left\langle\mathbf{2}, N_{\mathbf{2}}\right\rangle$ is $C_{\omega}$-substructure of any $\langle\mathbf{A}, N\rangle$, then we have the following lemma holds:

Lemma 2.10 (i) $\|u \in \hat{v}\|=\bigvee_{x \in v}\|u=\hat{x}\|$ for all $v \in \mathbf{V}$ and $u \in \mathbf{V}^{\langle\mathbf{A}, N\rangle}$,

(ii) $u \in v \leftrightarrow \mathbf{V}^{\langle\mathbf{A}, N\rangle} \vDash \hat{u} \in \hat{v}$ and $u=v \leftrightarrow \mathbf{V}^{\langle\mathbf{A}, N\rangle} \vDash \hat{u}=\hat{v}$,

(iii) for all $x \in \mathbf{V}^{\left\langle\mathbf{2}, N_{\mathbf{2}}\right\rangle}$ there exists a unique $v \in \mathbf{V}$ such that $\mathbf{V}^{\left\langle\mathbf{2}, N_{\mathbf{2}}\right\rangle} \vDash x=\hat{v}$,

(iv) for any formula negation-free formula $\psi\left(x_{1}, \cdots, x_{n}\right)$ and any $x_{1}, \cdots, x_{n} \in \mathbf{V}$, we have $\psi\left(x_{1}, \cdots, x_{n}\right) \leftrightarrow \mathbf{V}^{\left\langle\mathbf{2}, N_{2}\right\rangle} \vDash \psi\left(\hat{x_{1}}, \cdots, \hat{x_{n}}\right)$. Moreover for any restricted negation-free formula $\phi$, we have $\phi\left(x_{1}, \cdots, x_{n}\right) \leftrightarrow \mathbf{V}^{\langle\mathbf{A}, N\rangle} \vDash \phi\left(\hat{x_{1}}, \cdots, \hat{x_{n}}\right)$.

The proof of the last theorem is the same for intuitionistic case because we consider restricted negation-free formulas and it will be used to prove the validity of axiom Infinity.

\subsection{Validating axioms}

New, we are going to prove the validity of axioms of Set Theory and let us consider a fix model $\mathbf{V}^{\langle\mathbf{A}, N\rangle}$. Then

Extensionality Given $x, y \in \mathbf{V}^{\langle\mathbf{A}, N\rangle}$, then

$$
\begin{aligned}
& \|\forall z(z \in x \leftrightarrow z \in y)\|=\| \forall z\left((z \in x \rightarrow z \in y) \wedge(z \in y \rightarrow z \in x) \|=\bigwedge_{\substack{z \in \mathbf{V}\langle\mathbf{A}, N\rangle \\
x\|\rightarrow\| z \in}}(\|z \in y\|) \wedge \bigwedge_{z \in \mathbf{V}\langle\mathbf{A}, N\rangle}(\|z \in y\| \rightarrow\|z \in x\|) \leq \bigwedge_{\substack{z \in \operatorname{dom}(x) \\
\bigwedge_{z \in \operatorname{dom}(y)} \\
\|x=y\| .}}(\|z \in y\| \rightarrow\|z \in x\|) \leq \bigwedge_{z \in \operatorname{dom}(x)}(x(z) \rightarrow\|z \in y\|) \wedge \bigwedge_{z \in \operatorname{dom}(y)}(y(z) \rightarrow\|z \in x\|)=\right.
\end{aligned}
$$

Thus, we have $\|\forall x \forall y \forall z((z \in x \leftrightarrow z \in y) \rightarrow(x=y))\|$. On the other hand, for any $z \in$ $\mathbf{V}^{\langle\mathbf{A}, N\rangle}$ we infer that $\|x=y\| \wedge\|z \in x\| \leq\|z \in y\|$ and so, $\|x=y\| \leq\|z \in x\| \rightarrow\|z \in y\|$. Therefore, $\|\forall x \forall y((x=y) \rightarrow \forall z(z \in x \leftrightarrow z \in y))\|$. 
Pairing Let $u, v \in \mathbf{V}^{\langle\mathbf{A}, N\rangle}$ and consider the function $w=\{\langle u, 1\rangle,\langle v, 1\rangle\}$. Thus, we have that $\|z \in w\|=(w(u) \wedge\|z=u\|) \vee(w(v) \wedge\|z=v\|)=\|z=u\| \vee\|z=v\|=\|z=u \vee z=v\|$.

Powerset Assume $u \in \mathbf{V}^{\langle\mathbf{A}, N\rangle}$ and suppose $w$ a function such that $\operatorname{dom}(w)=\{f: \operatorname{dom}(u) \rightarrow$ $A: f$ function $\}$ and $w(x)=\|\forall y \in x(y \in u)\|$. Therefore,

$$
\|v \in w\|=\bigvee_{x \in \operatorname{dom}(w)}(\|\forall y \in x(y \in u)\| \wedge\|x=v\|) \leq\|\forall y \in v(y \in u)\| .
$$

On the other hand, given $v \in \mathbf{V}^{\langle\mathbf{A}, N\rangle}$ and consider the function $a$ such that $\operatorname{dom}(a)=\operatorname{dom}(u)$ and $a(z)=\|z \in u\| \wedge\|z \in v\|$. So, it is clear that $a(z) \rightarrow\|z \in v\|=1$ for every $z \in \operatorname{dom}(a)$, therefore

$$
\begin{aligned}
& \|\forall y \in v(y \in u)\|=\bigwedge_{y \in \operatorname{dom}(v)}(v(y) \rightarrow\|y \in u\|)=\bigwedge_{y \in \operatorname{dom}(v)}(v(y) \rightarrow(\|y \in u\| \wedge \\
& v(y))) \leq \bigwedge_{y \in \operatorname{dom}(v)}(v(y) \rightarrow a(y)) \leq \bigwedge_{y \in \operatorname{dom}(v)}(v(y) \rightarrow\|y \in a\|) \wedge \bigwedge_{z \in \operatorname{dom}(a)}(a(z) \rightarrow\|z \in v\|)= \\
& \|v=a\|
\end{aligned}
$$

Since $a(y) \leq\|y \in u\|$ for every $y \in \operatorname{dom}(a)$ then we have $\|\forall y \in a(y \in u)\|=1$. Now by construction we have that $a \in \operatorname{dom}(w)$ and so, $\|\forall y \in v(y \in u)\| \leq\|\forall y \in a(y \in u)\| \wedge \| v=$ $a\|=w(a) \wedge\| v=a\|\leq\| v \in w \|$.

Union Given $u \in \mathbf{V}^{\langle\mathbf{A}, N\rangle}$ and consider tha function $w$ with $\operatorname{dom}(w)=\bigcup_{v \in \operatorname{dom}(u)} \operatorname{dom}(v)$ and $w(x)=\bigvee_{v \in A_{x}} v(x)$ where $A_{x}=\{v \in \operatorname{dom}(u): x \in \operatorname{dom}(v)\}$. Then, $\|y \in w\|=\bigvee_{x \in \operatorname{dom}(w)}(\| x=$ $\left.y \| \wedge \bigvee_{v \in A_{x}} v(x)\right)=\bigvee_{x \in \operatorname{dom}(w)} \bigvee_{v \in A_{x}}(\|x=y\| \wedge v(x))=\bigvee_{v \in \operatorname{dom}(u)} \underset{x \in \operatorname{dom}(v)}{\bigvee}(\|x=y\| \wedge v(x))=$ $\|\exists v \in u(y \in v)\|$.

Separation Given $u \in \mathbf{V}^{\langle\mathbf{A}, N\rangle}$ and suppose $\operatorname{dom}(w)=\operatorname{dom}(u)$ and $w(x)=\|x \in u\| \wedge\|\phi(x)\|$ then $\|z \in w\|=\bigvee_{x \in \operatorname{dom}(w)}(\|y \in w\| \wedge\|\phi(y)\| \wedge\|y=z\|) \leq \bigvee_{x \in \operatorname{dom}(w)}(\|\phi(z)\| \wedge\|y=z\|)$

$$
\begin{gathered}
\text { Besides, }\|\phi(z)\| \wedge\|y=z\|=\bigvee_{y \in \operatorname{dom}(u)}(u(y) \wedge\|z=y\| \wedge\|\phi(z)\|) \\
\leq \bigvee_{y \in \operatorname{dom}(u)}(\|y \in u\| \wedge\|z=y\| \wedge\|\phi(y)\|)=\bigvee_{y \in \operatorname{dom}(u)}(w(y) \wedge\|z=y\|)=\|z \in w\| .
\end{gathered}
$$

Empty set Note that $\|u=u\|=1$ for all $u \in \mathbf{V}^{\langle\mathbf{A}, N\rangle}$ and then, $\|\neg(u=u)\| \in N_{1}$. Therefore, let us consider a function $w \in \mathbf{V}^{\langle\mathbf{A}, N\rangle}$ such that $u \in \operatorname{dom}(w)$ and $\operatorname{ran}(w) \subseteq\{\|\neg(u=u)\|\}$, then it is clear that $\|u \in w\|=\bigvee_{x \in \operatorname{dom}(w)}(w(x) \wedge\|u=x\|)=\|\neg(u=u)\|$.

Infinity Assume the formula $\psi(x)$ is $\emptyset \in x \wedge \forall y \in x\left(y^{+} \in x\right)$. Then, the axiom in question is the sentence $\exists x \psi(x)$. Now, it is clear that the negation-free formula $\emptyset \in x \wedge \forall y \in x\left(y^{+} \in x\right)$ is restricted and certainly $\psi(\omega)$ is true. Hence, by Lemma 2.10 (iv), we get $\|\psi(\hat{\omega})\|=1$, and so, $\|\exists x \psi(x)\|=1$.

Collection Given $u \in \mathbf{V}^{\langle\mathbf{A}, N\rangle}$ and $x \in \operatorname{dom}(u)$ there exists by Axiom of Choice some ordinal $\alpha_{x}$ such that $\bigvee_{y \in \mathbf{V}^{\langle\mathbf{A}, N\rangle}}\|\phi(x, y)\|=\bigvee_{y \in \mathbf{V}_{\alpha_{x}}^{\langle\mathbf{A}, N\rangle}}\|\phi(x, y)\|$. For $\alpha=\left\{\alpha_{x}: x \in \operatorname{dom}(u)\right\}$ and $v$ the function with domain $\mathbf{V}^{\langle\mathbf{A}, N\rangle}$ and range $\{1\}$, we have 


$$
\begin{aligned}
& \|\forall x \in u \exists y \phi(x, y)\|=\bigwedge_{x \in \operatorname{dom}(u)}\left(u(x) \rightarrow \bigvee_{y \in \mathbf{V} \backslash \mathbf{A}, N\rangle}\|\phi(x, y)\|\right)=\bigwedge_{x \in \operatorname{dom}(u)}(u(x) \rightarrow \\
& \left.\bigvee_{y \in \mathbf{V}_{\alpha}^{\langle\mathbf{A}, N\rangle}}\|\phi(x, y)\|\right)=\bigwedge_{x \in \operatorname{dom}(u)}(u(x) \rightarrow\|\exists y \in v \phi(x, y)\|)=\|\forall x \in u \exists y \in v \phi(x, y)\| \leq \\
& \|\exists w \forall x \in u \exists y \in w \phi(x, y)\| .
\end{aligned}
$$

Induction Let us suppose $x \in \mathbf{V}^{\langle\mathbf{A}, N\rangle}$, we are going to prove by induction on the well-founded relation $y \in \operatorname{dom}(x)$ and assume that $a=\|\forall x[(\forall y \in x \psi(y)) \rightarrow \psi(x)]\|$. On the other hand, assume that $a \leq\|\psi(y)\|$ for every $y \in \operatorname{dom}(x)$. So, it is clear that $a \leq \bigwedge_{y \in \operatorname{dom}(x)}\|\psi(y)\| \leq$ $\bigwedge_{y \in \operatorname{dom}(x)}(x(y) \wedge\|\psi(y)\|)=\|\forall y \in x \psi(y)\|$. But $a \leq\|(\forall y \in x \psi(y))\| \rightarrow\|\psi(x)\|$. Therefore, $a \leq[\|(\forall y \in x \psi(y))\| \rightarrow\|\psi(x)\|] \wedge\|\forall y \in x \psi(y)\| \leq\|\psi(x)\|$ as required.

Now, from the above proofs, we have proved the following theorem

Theorem 2.11 Let $\langle\mathbf{A}, N\rangle$ be complete $C_{\omega}$-structure such that $\mathbf{V}^{\langle\mathbf{A}, N\rangle}$ satisfies Leibniz's Law, then the all set-theoretic axioms of $Z F C_{\omega}$ axioms are valid in $\mathbf{V}^{\langle\mathbf{A}, N\rangle}$.

In this note, we present non-algebraic-valued models for a paraconsistent set theory showing Leibniz' Law is the only obstacle to getting full set theory. As a by product, we exhibit models for a full paraconsistent Set Theory theory rather than negation-free fragment improving the main result of Corollary 5.2 of [L-T 2015].

On the other hand, we have that this paraconsistent set theory the axiom of scheme Comprehension is not valid in our models; that is to say, it is enough to see that $\|\exists x \forall y(y \in x)\|=0$. In this setting, this paraconsistent set theory behaves similar to Löwe and Tarafder's paraconsistent set theory, see Theorem 6.3 of [L-T 2015].

\section{References}

Bell, J. (2005). Set theory. boolean valued models and independence proofs. Oxford Science Pubblications.

Bell, J. (2014). Intuitionistic set theory. College Publications.

da Costa, N. (1963). Sistemas formais inconsistentes. Habilitation thesis. Habilitation thesis, Universidade Federal do Paraná, Curitiba, Brazil.

Fidel, M. (1977). The decidability of the calculi $C_{n}$. Reports on Mathematical Logic, pages $31-40$.

Löwe, B. and Tarafder, S. (2015). Generalized algebra-valued models of set theory. Review of Symbolic Logic. 8(1), 192-205.

H. Rasiowa (1974) An algebraic approach to non-clasical logics, Studies in logic and the foundations of mathematics, vol. 78. North-Holland Publishing Company, Amsterdam and London, and American Elsevier Publishing Company, Inc., New York. 